\title{
Spatial distribution of HIV/AIDS in Yunnan province, People's Republic of China
}

\author{
Zhi-Hang Peng ${ }^{1}$, Yue-Jia Cheng', Kathleen H. Reilly²,3, Lu Wang ${ }^{2}$, Qian-Qian Qin ${ }^{2}$, \\ Zheng-Wei Ding ${ }^{2}$, Guo-Wei Ding ${ }^{2}$, Ke-Qin Ding ${ }^{1}$, Rong-Bin Yu ${ }^{1}$, Feng Chen ${ }^{1}$, Ning Wang ${ }^{2}$ \\ ${ }^{1}$ Department of Epidemiology and Biostatistics, School of Public Health, Nanjing Medical University, Nanjing \\ 210029, People's Republic of China; ${ }^{2}$ National Center for AIDS/STD Control and Prevention, Chinese Center \\ for Disease Control and Prevention, Beijing 102206, People's Republic of China; ${ }^{3}$ Tulane University Health \\ Sciences Center, School of Public Health and Tropical Medicine, New Orleans, LA 70112, USA
}

\begin{abstract}
Risk maps for the geographical distribution of human immunodeficiency virus (HIV) and the acquired immune deficiency syndrome (AIDS) are needed for the direction of HIV prevention interventions. Our study, based on county-level data on the numbers of HIV/AIDS patients in the Yunnan province, People's Republic of China, applied trend surface analysis and spatial autocorrelation analysis to demonstrate the geographical distribution of HIV-positive patients in the province. The case load of HIV was found to be most severe in the central-west region of the province. While Kunming county was shown to be negatively correlated with its surrounding counties, many high-burden counties are surrounded by other counties with similar case numbers. We conclude that intervention efforts in Yunnan province should concentrate on the western and northeast regions, targeting the hotspots of infection.
\end{abstract}

Keywords: HIV/AIDS, geographical information systems, trend surface analysis, spatial autocorrelation analysis, People’s Republic of China.

\section{Introduction}

Studies on the human immunodeficiency virus (HIV) and the acquired immune deficiency syndrome (AIDS) in the People's Republic of China (P. R. China) and abroad have primarily focused on aspects such as the molecular features and transmission characteristics of the disease (Andreas, 2010), the efficacy of therapies and vaccine development (Wayne, 2010), estimating and forecasting epidemiological trends ( $\mathrm{Hu}$ et al., 2006), exploring the contribution of other diseases on HIV transmission (van Houdt et al., 2010) and promoting patient advocacy. However, the number of published studies on the spatial distribution of HIV/AIDS is more limited. The current study examines the spatial distribution of HIV/AIDS in the Yunnan province, P. R. China in an attempt to find a relationship between HIV/AIDS prevalence and its geographical distribution.

Corresponding author:

Ning Wang

National Center for AIDS/STD Control \& Prevention

Chinese Center for Disease Control \& Prevention

155 Changbai Road, Changping District, Beijing 102206

People's Republic of China

Tel. +8610 5890 0906; Fax 8658900906

E-mail: wangnbj@163.com
Yunnan province is located in the southern part of P. R. China, bordering Vietnam, Lao People's Democratic Republic and Myanmar (Fig. 1). The first Chinese case of HIV/AIDS was discovered in 1985 in Yunnan province (Lu et al., 2004). At that time, the HIV epidemic was primarily restricted to injectiondrug users in P. R. China, which now has one of the largest populations of injection-drug users in the world, $12 \%$ of whom are estimated to be HIV positive (Yan et al., 2010). Although the epidemic has shifted

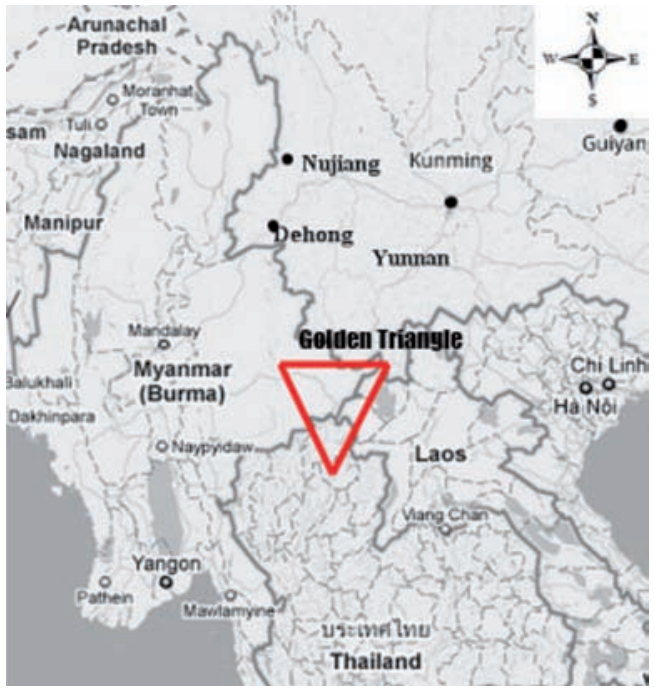

Fig. 1. Yunnan province in P.R. China and neighbouring countries with the location of the "Golden Triangle". 
from transmission through injection of illegal drugs to transmission through commercial sex workers ( $\mathrm{Lu}$ et al., 2008), the total number of injection-drug users in Yunnan province has increased during the last 20 years (Jia et al., 2010).

In 2007, six of P. R. China's 32 provinces accounted for $80.5 \%$ of all HIV/AIDS infections and Yunnan had the largest number, both of full-blown AIDS cases and deaths due to the infection (Wang et al., 2009b). The large number of sex workers and injection-drug users in Yunnan province contribute to the high prevalence of the infection (Wang et al., 2009a). In 2010, the World Health Organization (WHO) announced that the cumulatively reported cases of HIV/AIDS in P. R. China had reached 370,393 (Disease Control Bureau, Ministry of Health, 2010). An assessment conducted by the Chinese Ministry of Health, the Joint United Nations Programme on HIV/AIDS (UNAIDS) and WHO estimated that there were approximately 48,000 new HIV infections in 2009 in P. R. China (Barouch, 2008; Peng et al., 2010).

Since there is a geographic indicator related to the distribution of almost $80 \%$ of all diseases (Xu and Zhang, 2003), a better understanding of the disequilibrium and distribution of communicable diseases would provide decision-support for the allocation of medical resources and provide effective guidance for public health interventions (Antunes et al., 2002; Moreira et al., 2007). The current study investigated the spatial distribution of HIV/AIDS in Yunnan province to facilitate prevention and control. The approach taken was to analyse the spatial distribution of HIV/AIDS in the counties of Yunnan province and describe the geographical characteristics of HIV/AIDS using geographical information systems (GIS) and spatial statistics.

\section{Material and methods}

The sample studied was composed of HIV/AIDS cases from all of Yunnan province's 125 counties. Data were obtained from the HIV/AIDS sentinel surveillance system of the Chinese Center for Disease Control and Prevention (China CDC) for the years 1991-2009. Demographic data came from the National Statistics Department. The full range of HIV/AIDS infections per county $(5-5,420)$ was divided into five levels based on the number of HIV/AIDS cases observed, or by the "Natural Breaks" method, which is used to find a partition capable of achieving the minimum difference between the samples in the same section, and the maximum difference between samples in various sections (Wang, 2006).
The ArcGIS software, version 9.3 (ESRI; Redlands, CA, USA) was used to map the numbers of HIV/AIDS cases by county. The data were subjected to statistical analyses using four different approaches as described below.

\section{Trend surface analysis}

With the objective of highlighting geographical anomalies and describe scattered observations, the global-fit approach was used to produce a threedimensional projection of the geographical distribution of the number of cases (Fig. 2). GIS was applied to locate each HIV/AIDS case, deriving the coordinates expressing the original residential locations of the cases in the format of latitude (denoted as X) and longitude (denoted as Y). To minimise the squared surface deviations, the coefficients of the polynomial functions were calculated by the ordinary least square (OLS) technique (Wang, 2006). With the number of HIV/AIDS cases in each node of the projected grid matrix represented by $Z$ and letting the $X$ axis point east and the $\mathrm{Y}$ axis south, directional trend curves of the case locations were produced.

\section{Spatial autocorrelation}

According to Tobler's first law of geography (Tobler, 1970), "everything is related to everything else, but close things are more related than distant things". With that in mind, spatial autocorrelation was used to analyse the distribution of HIV/AIDS in Yunnan province with the aim of detecting infection clusters and the factors affecting the distribution. Where no spatial autocorrelation existed, the cases were considered to be distributed at random. Moran's I (Moran, 1950) and the Getis statistics, available as an ArcGIS application, was used as measures of autocorrelation.

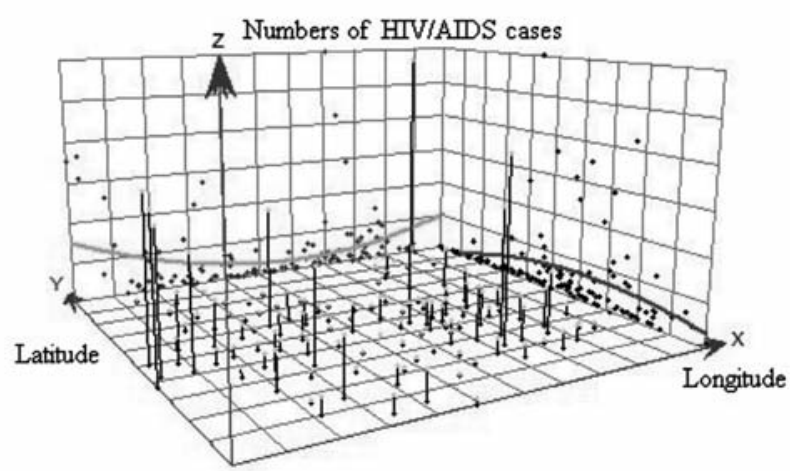

Fig. 2. Trend surface analysis map for HIV/AIDS in Yunnan province, P. R. China. 


\section{General spatial autocorrelation}

Assuming that the counties in the province did not differ from each other (He et al., 2008), autocorrelation was applied to analyse the province as a whole. Using the aggregation of the whole province made it possible to make out whether or not it belonged to a high-risk group of provinces. The general Moran's I coefficient was calculated as:

$$
\mathrm{I}=\frac{n}{\sum_{i=1}^{n} \sum_{j=1}^{n} w_{i j}} \times \frac{\sum_{i=1}^{n} \sum_{j=1}^{n} w_{i j}\left(x_{i}-\bar{x}\right)\left(x_{j}-\bar{x}\right)}{\sum_{j=1}^{n}\left(x_{i}-\bar{x}\right)}
$$

where $n$ is the number of spatial units (counties), $x_{i}$ and $x_{j}$ the observations from unit $i$ to unit $j$ about the phenomenon $X ; w_{i j}(d)$ represents the adjacent weight matrix from the distance $d$. As for regional data, if the unit $i$ was adjacent to the unit $j$, then $w_{i j}(d)$ would be 1 , otherwise, it would be 0 . This study assumed a normal distribution of randomly dispersed cases, i.e. if $\mathrm{P}<0.05$, than Moran's $I \neq 0$ meaning that the area had a cluster of HIV infections. Moran's I index makes it possible to decide whether there is an autocorrelation by the counties, i.e. if $I>0$, then the HIV/AIDS is positively autocorrelated.

The general Getis index $\left(\mathrm{G}_{i}\right)$, based on the same factors was calculated as:

$$
\mathrm{G}_{i}(d)=\frac{\sum_{i=1}^{n} \sum_{i=1, j \neq i}^{n} w_{i j}(d) x_{i}-x_{j}}{\sum_{i=1}^{n} \sum_{j=1, j \neq i}^{n} x_{i}-x_{j}}
$$

The $\mathrm{G}_{i}$ was compared with the critical value of $Z$, i.e. $P<0.05$ indicates a high spatial autocorrelation between counties. The $\mathrm{G}_{i}$ makes it possible to find the counties with high-value or low-value correlations, i.e. if $\mathrm{G}>0$, then the counties highlighted all have a HIV/AIDS incidence above the average level of the province overall and they are correlated.

\section{Local spatial autocorrelation}

Given that the HIV/AIDS epidemic may vary geographically throughout the province, e.g. due to source of infection, mode of transmission, population characteristics, and behavioural distinctions, the counties may not always exhibit the same features as neighbouring counties. Thus, the results of general spatial autocorrelation could be contrary to the results of the local spatial autocorrelation. The latter was used to examine whether there indeed are regional patterns involving specific areas in the province. As the results can indicate specific autocorrelation among high-risk counties and the focal points of HIV/AIDS, a local Moran's I (3) and a local G index (4) was calculated using the following equations:

$$
\begin{gathered}
I_{i}=\frac{n^{2}}{\sum_{i} \sum_{j} w_{i j}} \times \frac{\left(x_{i}-\bar{x}\right) \sum_{j} w_{i j}\left(x_{j}-\bar{x}\right)}{\sum_{j}\left(x_{j}-\bar{x}\right)^{2}} \\
\mathrm{G}_{i}(d)=\frac{\sum_{i=1}^{n} \sum_{j=1, j \neq i}^{n} w_{i j}(d) x_{j}}{\sum_{i=1}^{n} \sum_{j=1, j \neq i}^{n} x_{i}} \quad i \neq j
\end{gathered}
$$

\section{Results}

Figure 3 shows the distribution of HIV/AIDS in Yunnan province, stratifed by county. More than half of the counties investigated were characterised by HIV/AIDS infections at the lowest level of prevalence (5-263 cases), presented as white in the map, while counties with greater numbers of HIV infections are in darker colour. Only nine counties were shown to belong to the two top levels of infection (Table 1). On average, the number of cases in the south was higher than those in the north. However, the very most affected counties were primarily situated in the far west and east of the province.

\section{Trend surface analysis}

Figure 2 illustrates the analysis indicating the geographical variation of HIV/AIDS in Yunnan province. When the distribution is observed longitudinally it can

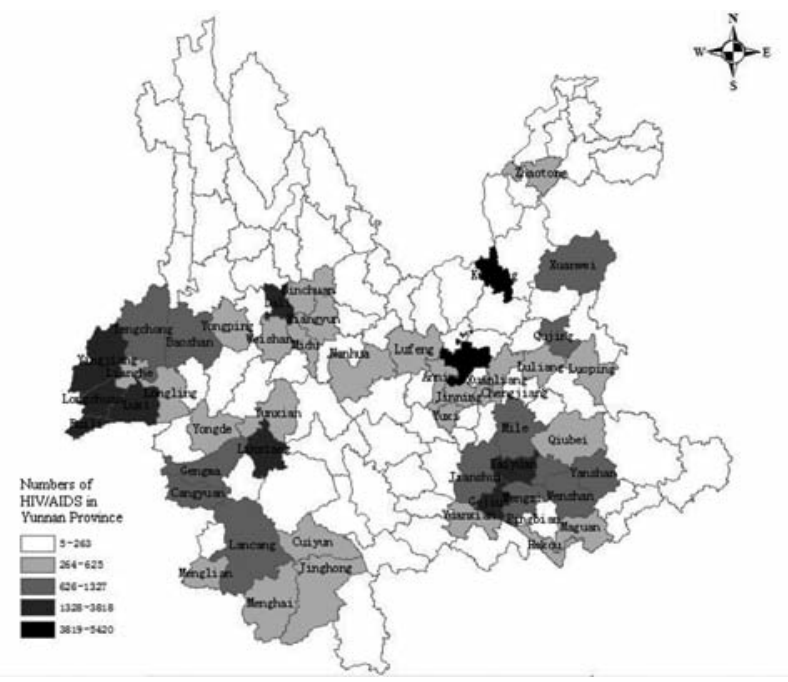

Fig. 3. The general spatial autocorrelation for HIV/AIDS in Yunnan province, P. R. China. 
Table 1. The number of HIV/AIDS cases in the nine counties of Yunnan province, P. R. China with the highest number of cases.

\begin{tabular}{ll}
\hline County & Cases \\
\hline Linxiang & 1,947 \\
Luxi & 2,303 \\
Dali & 2,467 \\
Kaiyuan & 2,641 \\
Longchuan & 2,803 \\
Ruili & 3,187 \\
Yingjiang & 3,304 \\
Gejiu & 3,818 \\
Kunming & 5,420 \\
\hline
\end{tabular}

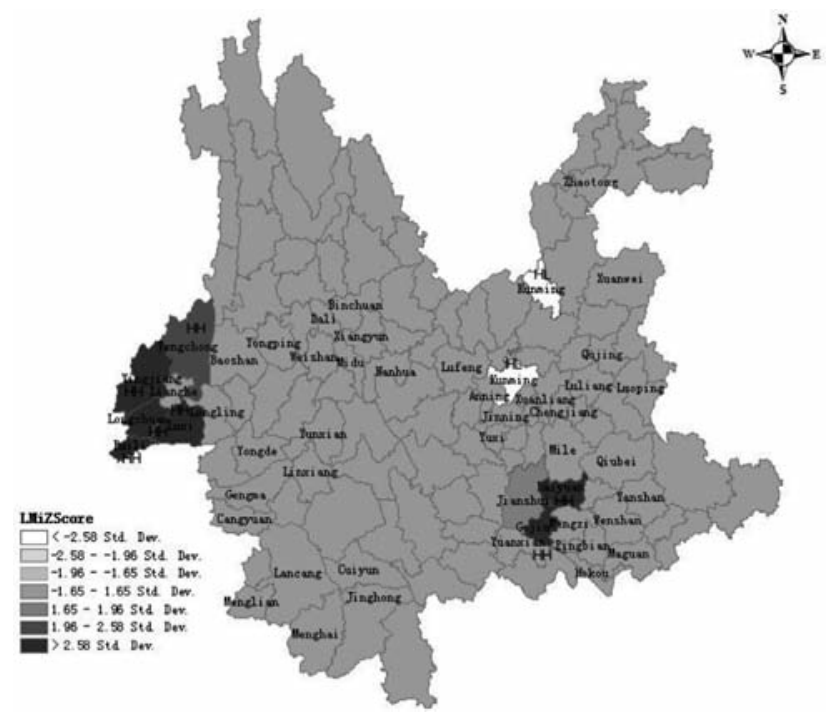

Fig. 4. Predictive risk map of HIV/AIDS in Yunnan province, P.R. China. be seen that the number of HIV/AIDS cases increases from the south towards the centre of the province and then decreases towards the north. The variation along the latitudinal axis shows that there are more HIV/AIDS cases in the west compared with the situation in the east. As can be seen from depicted curves on the "sides" of the projection, the number of cases appears to vary smoothly in these two directions. Although schematic, the findings from the trend surface analysis corroborate the risk-map, and the outcomes of Moran's $I=0.14(\mathrm{P}=0.002)$ and the Getis statistics $=0.000001(\mathrm{P}=0.08)$ confirm that the location of the HIV/AIDS cases in Yunnan province are positively autocorrelated.

\section{Local spatial analysis}

The local Moran's I map of the distribution of the HIV/AIDS cases for Yunnan province, stratifed by county, classified into seven levels, is shown in Figure 4 with Table 2 listing all counties showing a statistically significant autocorrelation $(\mathrm{P}<0.05)$. Eight counties were considered to be positively autocorrelated, i.e. they were not only demonstrated as high-burden counties but they were also shown to be surrounded by counties with large numbers of cases. The Longchuan and Ruili counties had the highest indexes (Table 2). Several other counties, i.e. Yingjiang, Luci, Kaiyuan and Gejiu, also showed high positive correlations $(\mathrm{P}<0.00001)$. However, with a local Moran's $I$ of -0.00043 , Kunming was negatively autocorrelated, meaning that this county has a large number of cases but is surrounded by counties with low numbers of cases. The highly correlated counties were concentrated in two parts of Yunnan province: in the far west and the north-east (Fig. 4). The Getis statistics confirmed the autocorrelative findings of local Moran's I

Table 2. The result of local Moran's I for HIV/AIDS in Yunnan province, P. R. China.

\begin{tabular}{lcccc}
\hline County & LMi* $^{*}$ & LMi Z score & LMi P value & Correlation \\
\hline Tengchong & 0.00005 & 2.00841 & 0.04460 & High-high \\
Kunming & -0.00043 & -7.07961 & $<0.00001$ & High-low \\
Yingjiang & 0.00032 & 9.87997 & $<0.00001$ & High-high \\
Longchuan & 0.00054 & 13.99940 & $<0.00001$ & High-high \\
Luxi & 0.00031 & 7.41989 & $<0.00001$ & High-high \\
Ruili & 0.00033 & 12.42070 & $<0.00001$ & High-high \\
Kaiyuan & 0.00026 & 6.17016 & $<0.00001$ & High-high \\
Gejiu & 0.00023 & 4.97351 & $<0.00001$ & High-high \\
\hline
\end{tabular}

\footnotetext{
* Local Moran's I
} 
(Table 3). The counties were all positively spatially autocorrelated and are thus hotspots for HIV/AIDS in Yunnan province.

\section{Limitations}

The real distribution of the HIV/AIDS epidemic in Yunnan province could deviate from that estimated by statistical methods but only slightly so. The major risk factors contributing to the transmission of HIV/AIDS in Yunnan province (injection-drug use and commercial sex), may well be geographically related with the distribution of the cases of HIV/AIDS found. However, the paucity of data on the distribution of high-risk behaviour, due to the fact that discrimination of the individuals most likely to contribute the most to the HIV/AIDS epidemic make them avoid HIV testing, precluded its full consideration here.

Our data relies on official surveillance and it is possible that some counties may underreport the number

Table 3. Local Getis statistic for HIV/AIDS in Yunnan province, P. R. China.

\begin{tabular}{lcc}
\hline County & Gi Z score & Gi P value \\
\hline Tengchong & 2.53018 & 0.01140 \\
Yingjiang & 4.29077 & 0.00002 \\
Lianghe & 3.85872 & 0.00011 \\
Longchuan & 5.38545 & $<0.00001$ \\
Luxi & 4.02644 & 0.00006 \\
Ruili & 4.84998 & $<0.00001$ \\
Kaiyuan & 2.73075 & 0.00632 \\
Gejiu & 2.64524 & 0.00816 \\
Mengzi & 2.65902 & 0.00784 \\
Bingbian Miaozu Zizhixian & 2.50390 & 0.01228 \\
\hline
\end{tabular}

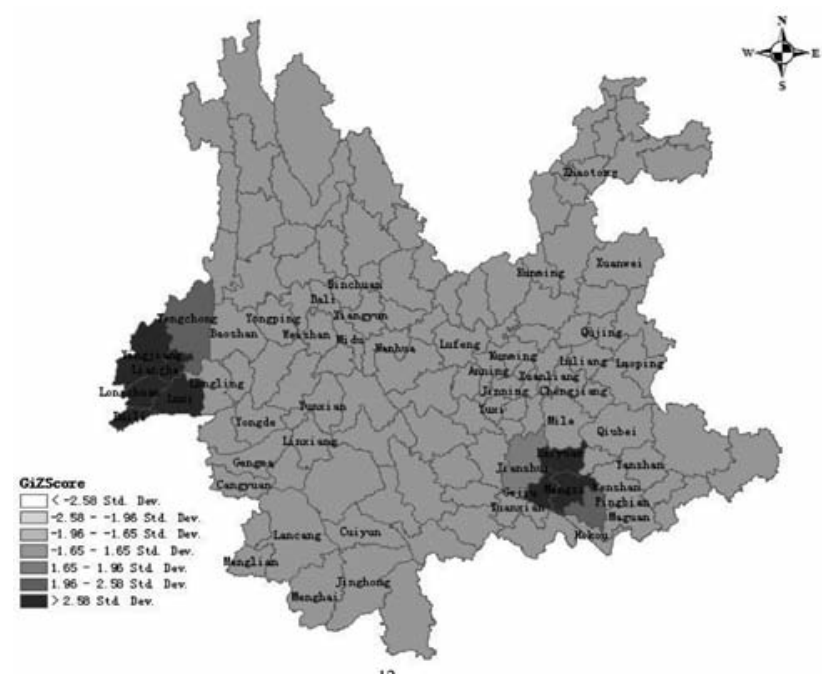

Fig. 5. Local Moran's I analysis map for autocorrelation. of cases for various reasons. There are also many cases of HIV-infected people who have not (yet) been identified, or who were identified during the time covered by the study. However, this situation is currently being rectified since the Ministry of Health has increased case finding in recent years and the number of sentinel surveillance sites has been increased from 845 in 2007 to 1,995 in 2009 .

\section{Discussion}

The location of Yunnan province adjacent to the "Golden Triangle" (Fig. 1) has opened the door for expanding HIV/AIDS transmission into the province. In addition, the "social cultural distance" can be as strong as geographic distance (Bao, 2010). Borderland counties gradually develop a multicultural society, which includes both countries and leads to small trade and even intermarriage. Frontier trade and travel contribute to growing numbers of people, referred to as "floating population", being attracted to illegal activities such as sexual sale, drug smuggling, trading with uncontrolled blood products, etc. The new environment, supported by improved communication, paves the way for a strengthening correlation of drug use and sex trade with HIV/AIDS incidence.

The trend surface analysis and the risk maps produced indicate that the epidemic is most severe in the far west and the north-eastern regions. The finding that half of the "hot spots" are located in the part of Yunnan that borders Myanmar is hardly surprising as its drug production accounts for $80 \%$ of that of the whole "Golden Triangle" (Beyrer et al., 2010), and the connection between HIV/AIDS and the use of intravenous drug use is well known (Booth et al., 1993; Beyrer et al., 2010). The same goes for the other half of the "hot spots" in the south-east, as Yunnan's main transport artery goes through this area, not only bringing in goods but also the HIV/AIDS epidemic. Indeed, the development of transportation has promoted the growth and spread of the "floating population" (Liu et al., 2005), and studies by Bao (2010) shows that HIV/AIDS cases diffuse along the main transport lines into surrounding areas. However, in the far south-western corner of Yunnan province, there are relatively small numbers of HIV/AIDS cases, exemplified by Diqing county (92 cases) and Nujiang county (296 cases). Despite being located near high-risk counties (i.e. Dehong and Baoshan), historical reasons and their particular environment contribute to keeping these counties less prone to the influx of modern life. Thus, minority people with conservative popular cus- 
toms, living in areas with limitation transport possibilities and less developed communication, show low incidence of HIV/AIDS. In fact, the presence of Miaozu and Yizu, two such minority groups, which dominate the surroundings of Kunming, may well explain the negative autocorrelation noted with regard to this county, despite its standing as one of the biggest and most thriving cities in Yunnan province. Indeed, the economic development in Kunming can act as a magnet, not only for people seeking a better future, but also attracting large crowds of "floating population", who would rather stay than diffusing into less populous areas characterised by ethnically related people with a strong affinity to age-old popular customs.

In conclusion, Yunnan province is at high risk for further growth and dissemination of the HIV/AIDS epidemic. It is important to strengthen intervention efforts concentrating on the far west and the north-eastern regions targeting the HIV "hot spots".

\section{Acknowledgements}

This work was supported in part by "National S\&T Major Project Foundation of China" (2008ZX10001-003), National Natural Science Foundation of China (81001288).

\section{References}

Antunes JL, Frazão P, Narvai PC, 2002. Spatial analysis to identify differentials in dental needs by area-based measures. Community Dent Oral Epidemiol 30, 133-142.

Bao G, 2010. Study on the succession mechanism of HIV/AIDS and the counter-measures in Yunnan province. Northwest Population J 31, 105-107.

Barouch DH, 2008. Challenges in the development of an HIV-1 vaccine. Nature 455, 613-619.

Beyrer C, Wirtz AL, Baral S, Peryskina A, Sifakis F, 2010. Epidemiologic links between drug use and HIV epidemics: an international perspective. J Acquir Immune Defic Syndr 1, 10-16.

Booth RE, Watters JK, Chitwood DD, 1993. HIV risk-related sex behaviors among injection drug users, crack smokers, and injection drug users who smoke crack. Am J Public Health 83, 1144-1148.

He Z, Han S, Cui D, Zhang Z. 2008. Discussion on statistic of spatial autocorrelation analysis. Chinese J Schistosom Control 20, 315-317.

Hu Z, Qin X, Zhu MZ, Yang S, Zhang XJ, 2006. Epidemiological characteristics of HIV/AIDS in west China. Int J STD AIDS 17, 324-328.

Jia M, Luo H, Ma Y, Wang N, Smith K, Mei J, Lu R, Lu J, Fu
L, Zhang Q, Wu Z, Lu L, 2010. The HIV epidemic in Yunnan province, China, 1989-2007. J Acquir Immune Defic Syndr 53, 34-40.

Liu D, Ying F, Luo H, 2005. The macroscopic mechanism analysis about the spread HIV/AIDS in Yunnan province, China. Chin J AIDS STD 11, 267-271.

Lu L, Jia M, Ma Y, Yang L, Chen Z, Ho DD, Jiang Y, Zhang L, 2008. The changing face of HIV in China. Nature 455, 609-611.

Lu L, Jia MH, Zhang XB, Luo HB, Ma YL, Fu LR, Lu JY, 2004. Analysis for epidemic trend of acquired immunodeficiency syndrome in Yunnan province of China. Chin J Prev Med 5, 309-312.

Moran PAP, 1950. Notes on continuous stochastic phenomena. Biometrika 37, 17-33.

Moreira RF, Nico LS, Tomita NE, 2007. The relation between space and collective oral health: for a georeferenced epidemiology. Cien Saude Colet 12, 275-284.

Ministry of Health, 2010. The achievements of AIDS prevention and control work in China. The Disease Control Bureau 201011-29. http://www.moh.gov.cn/publicfiles/business/htmlfiles/mohjbyfkzj/s3586/201011/49893.htm.

Peng Z, Wang L, Yu R, Ding G, Yu H, Chen F, Wang N. 2010. The Asia epidemic model of AIDS and its' application to the AIDS epidemic forecast in China. Chinese J Prev Med 44, 97-100.

Plückthun A, 2010. HIV: antibodies with a split personality. Nature 467, 537-538.

Tobler WR, 1970. A computer movie simulating urban growth in the Detroit region. Econ Geogr 46, 234-240.

van Houdt R, Bruisten SM, Geskus RB, Bakker M, Wolthers KC, Prins M, Coutinho RA, 2010. Ongoing transmission of a single hepatitis $\mathrm{B}$ virus strain among men having sex with men in Amsterdam. J Viral Hepat 1, 108-114.

Wang F, 2006. Quantitative Methods and Applications in GIS. Taylor \& Francis Group

Wang H, Chen RY, Ding G, Ma Y, Ma J, Jiao JH, Wu Z, Sharp GB, Wang N, 2009a. Prevalence and predictors of HIV infection among female sex workers in Kaiyuan city, Yunnan province, China. Int J Infect Dis 13, 162-169.

Wang L, Wang N, Wang L, Li D, Jia M, Gao X, Qu S, Qin Q, Wang Y, Smith K, 2009b. The 2007 estimates for people at risk for and living with HIV in China: progress and challenges. J Acquir Immune Defic Syndr 50, 414-420.

Wayne C, 2010. Accelerating HIV vaccine development. Nature 464, 161-162.

$\mathrm{Xu} \mathrm{D}$, Zhang Z, 2003. The geographic information system and remote sensing in epidemiology. Chin J Epidemiol 24, 251-252. Yan L, Duan S, Gong Y, Yang Y, Xiang L, Ye R, Pang L, Liu E, Wu Z. 2010. An evaluation study on HIV risk behaviors and infections in four detoxification centers in Yunnan Province. Chinese Journal of AIDS \& STD 16, 149-151. 